\title{
Veículos aéreos não tripulados e sensores remotos: ferramentas para o gerenciamento dos recursos florestais
}

\author{
Igor Viana Souza $^{a} \oplus$, Victor Braga Rodrigues Duarte ${ }^{a} \oplus$, Francisca de Cássia Silva da Silva ${ }^{a} \odot$, \\ Marcos Cardoso Silva ${ }^{\mathrm{a}}$, Hygor Gomes de Almeida Sousa ${ }^{\mathrm{a}} \oplus$, Jhennyfer Nayara Nogueira Gomes ${ }^{\mathrm{a}} \odot$, \\ Marcos Giongo ${ }^{\mathrm{a}}{ }^{\odot}$, Antônio Carlos Batista ${ }^{\mathrm{b}} \odot$ \\ ${ }^{a}$ Universidade Federal do Tocantins, Brasil \\ ${ }^{\mathrm{b}}$ Universidade Federal do Paraná, Brasil \\ *Autor correspondente (igor.souza@uft.edu.br)
}

\section{N F O}

\section{Keywords}

remote sensing

UAVs

vegetation

\begin{abstract}
A B S T R A C T
Unmanned aerial vehicle and remote sensors: tools for the management of forest resources

The objective of this work is to describe, through the literature review, the use of remote sensors integrated to unmanned vehicles (UAV). The types of sensors and their operation are also described in addition to the processing methods and applications in forestry and protection against forest fires. The UAV was used for military activities, mainly for monitoring areas of difficult access, for human use. With the technological development of vehicles equipped with the Global Satellite Navigation System (GNSS) and minimization of sensors made possible to capture electromagnetic electrical energy reflected and transmitted by targets on the surface, being converted into traffic signals so that they can be stored or transmitted in real time. The data captured by the sensors are processed to be used in the process of using information from objects observed on the earth's surface to be used in the management of forest resources.
\end{abstract}

\section{R E S U M O}

O objetivo deste trabalho é descrever, através de revisão da literatura, o uso dos sensores remotos integrados aos Veículos Aéreos Não tripulados (VANT). Os tipos sensores e seu funcionamento também são apresentados além dos métodos de processamento e aplicações na silvicultura e na proteção contra incêndios florestais. O VANT foi utilizado inicialmente em atividades militares, principalmente para atender monitoramento de áreas de difícil acesso, consideradas inóspitas para o ser humano. Com o desenvolvimento tecnológico dos veículos equipados com Sistema Globais de Navegação por Satélite (GNSS) e miniaturização dos sensores possibilitaram a captação da energia eletromagnética refletida e transmitida pelos alvos na superfície, sendo posteriormente convertida em sinais elétricos de maneira que podem ser armazenadas ou transmitidas em tempo real. Os dados capturados pelos sensores são processados para serem utilizados no processo de aquisição de informações dos objetos observados na superfície terrestre para serem aplicados no gerenciamento dos recursos florestais. 


\section{INTRODUÇÃO}

A legislação pertinente (Circular de Informações Aéreas AIC N 21/10) define Veículo Aéreo Não Tripulado (VANT) como uma plataforma capaz de voar no espaço suborbital, fora do efeito de solo, projetado ou adaptado para não receber um piloto humano e que é operado por controle remoto ou de forma autônoma.

O VANT foi utilizado inicialmente para o uso militar, visando executar missões que oferenciam riscos ao ser humano. O desenvolvimento de recursos, como sensores remotos aclopados, mostrou-se ferramenta alternativa em atividades profissionais e em pesquisas de campo, tornandose aplicável em tarefas complexas e onerosas, particulamente na redução dos custos com a aquisição de imagens e equipamentos, garantindo maior flexibilidade de resolução temporal. Dessa forma, proporcionando uma otimização das atividades de campo (Raj et al., 2019).

Os primeiros estudos sobre o uso do VANT foram desenvolvidos por Przybilla e WesterEbbinghaus (1979), demonstrando sua eficácia, principalmente na inspenção de culturas, detecção de espécies daninhas, identificação de falhas no plantio, volumetria, biomassa e índices de vegetação.

Há uma variedade de VANTs e sensores que podem ser utilizados no gerenciamento dos recursos florestais, requerendo aos profissionais conhecimento em relação ao uso das novas ferramentas utilizadas, colaborando para o desenvolvimento de novas metodologias de gerenciamento dos recursos.

Dessa forma, o objetivo desta revisão é descrever o uso dos sensores remotos integrados aos Veículos Aéreos Não tripulados (VANT), assim como, comparar os VANTs de asa fixa e multirrotores. Os tipos de sensores e seu funcionamento também são apresentados além dos métodos de processamento e aplicações na silvicultura e controle de incêndios florestais.

\section{CATEGORIZAÇÃO DOS VEÍCULOS AÉREOS NÃO ŢRIPULADOS (VANT)}

Os VANTs são conhecidos principalmente pelas missões realizadas por meio de drones, termo que designa a categoria dos multirrotores, que realizam sua sustentação no ar por meio do empuxo das hélices, oferencendo versatilidade em sua decolagem e pouso vertical. Contudo, sua operacionalidade esbarra na baixa autonomia de voo, sendo adequados para cobrir pequenas áreas (Jorge e Inamasu, 2014) (Figura 1).

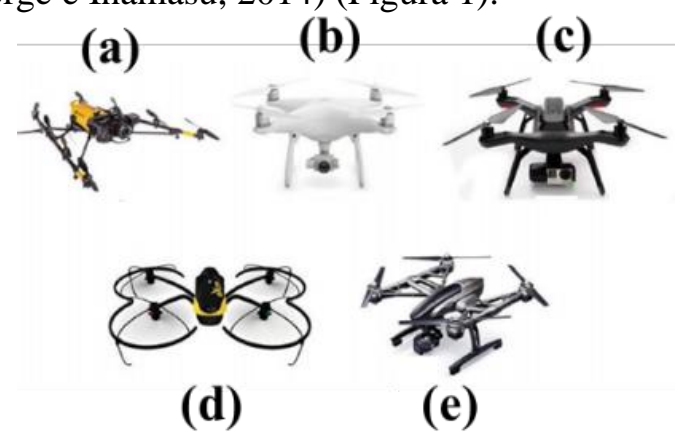

Fonte: adaptado de Pádua et al. (2017).

Figura 1 - Multirrotores comum no mercado: a) Topcon Falcon 8; b) DJI Phantom 4; c) 3DR SOLO Quadcopter; d) SenseFly eXom; e) Yuneec Typhoon.

Os melhores desempenhos, considerando-se a autossuficiência de voo, são verificados com os equipamentos de asas fixas, lançados manualmente ou por meio de uma rampa de lançamento. A plataforma é considerada como a mais adequada para o levantamento aéreo, necessitando de uma maior cobertura de área, bem como de uma área ampla para a aterrisagem (Pádua et al., 2017) (Figura 2).

(a)

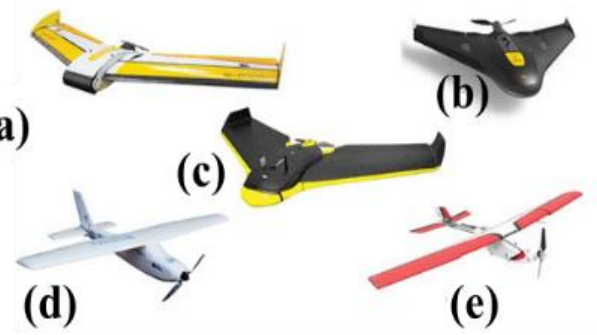

Fonte: adaptado de Pádua et al. (2017).

Figura 2 - Asas fixas disponíveis no mercado: (a) QuestUAV Q-Pod; (b) eBee SenseFly; (c) Trimble UX5; (d) MAVinci Sirius Pro; e (e) PrecisionHawk Lancaster.

Após a decolagem do VANT, o receptor do Sistema Global de Navegação por Satélite (GNSS) é responsável por direcionar o veículo para sua missão previamente planejada com as seguintes etapas: (1) plano de voo - realizada por meio de softwares ou aplicativos para smarthphones; (2) execução da missão - os sensores acoplados no veículo capturam automaticamente as imagens usando o GNSS como referência; (3) fim da missão - após o pouso do veículo as imagens são processadas em softwares com produtos desejados; (4) avaliação dos dados dos produtos por exemplo, quantificação de biomassa, 
volumetria e identificação de falhas em plantios (Hardin e Jensen, 2011).

\section{SISTEMA DE SENSORES REMOTOS EM VEÍCULOS AÉREOS NÃO TRIPULADO (VANTS)}

Os VANTs, por serem veículos leves, possuem capacidade limitada de peso a ser transportada, sendo necessário realizar mais de uma missão em um levantamento. Por outro lado, com o aperfecioamento dos sensores remotos, tornou-se possível adequar a capacidade de carga aos VANTs.

O uso dos sensores remotos acoplados em VANTs permite captar a energia eletromagnética refletida pelo alvo, sendo convertida em sinais elétricos de maneira que possa ser armazenada ou transmitida em tempo real, com o propósito de ser utilizada no processo de aquisição de informações dos objetos observados na superfície terrestre (Moraes, 2008).

$\mathrm{O}$ sistema de sensores remotos permitem gerar produtos, por exemplo, imagens de um objeto por meio de scanners, câmaras fotográficas ou em resultados na forma digital, captadas por sensores não imageadores, denomidados de espectrorradiômetros (Liu, 2015).

Os sensores podem ser classificados de acordo com a forma de captação da radiação eletromagnética, ativa ou passiva. Os sensores ativos possuem uma fonte de energia que incide sobre o objeto como, por exemplo, sensores LiDAR que emitem impulsos em forma de LASER armazenando informações em relação ao alvo. Os sensores passivos não necessitam de uma fonte de energia eletromagnética aclopada na plataforma. Um sensor fotográfico é exemplo de sensor passivo (Liu, 2015).

A capacidade do sensor fornecer informações detalhadas do alvo está relacionada a três tipos de resolução: espacial, espectral e radiométrica. A resolução espacial é a capacidade de distinguir objetos na superfície terrestre, sendo influenciada principalmente pela altura e posicionamento do sensor, quanto menor a resolução geométrica maior será o grau de detalhamento do objeto. Por exemplo, um sensor fotográfico nas dimensões de $1 / 2.3^{\prime}$ com resolução $4.000 \times 3.000$ pixels e distância focal de $4,73 \mathrm{~mm}$ em uma altura de 80 metros possui um Ground Sample Distance de 2,5 $\mathrm{cm} /$ pix, ou seja, objetos inferiores a esta dimensão não serão detectadas pelo sensor (Souza et al., 2018) (Figura 3).

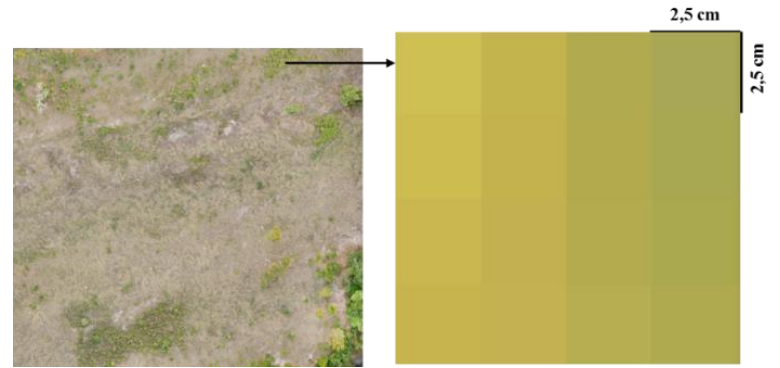

Figura 3 - Exemplificação do resolução geométrica de um sensor fotográfico nas dimensões de 1/2.3" com resolução 4.000 x 3.000 pixels e distância focal de $4,73 \mathrm{~mm}$.

A qualidade da resolução espectral está relacionada à largura espectral que o sensor opera, $\mathrm{e}$, desse modo, quanto maior for o número de medidas em um determinado intervalo do comprimento de ondas, melhor será a sua resolução espectral. Da mesma forma, a resolução radiométrica está relacionada à capacidade do sensor em distinguir e registrar a energia captada, quanto maior a capacidade do sistema, melhor será a distinição dos elementos que compoem a cena como, por exemplo, vegetação, hidrologia e solo (Moraes, 2008).

\section{Sensores Red, Green and Blue (RGB)}

Os sensores $R G B$ são câmeras fotográficas capazes de capturar imagens perceptíveis ao olho humano operando na faixa espectral do vísivel. A sensação das cores captadas pelo sistema visual humano é produzido pela luz associada a diferentes comprimentos de ondas, conforme a distribuição de cores apresentada na Tabela 1 (Ponzoni et al., 2015).

Tabela 1 - Relação entre cores e comprimento de onda.

\begin{tabular}{cc}
\hline Cor & Intervalo de comprimento de onda \\
\hline Violeta & $0,380-0,450 \mu \mathrm{m}$ \\
Azul & $0,450-0,495 \mu \mathrm{m}$ \\
Verde & $0,495-0,570 \mu \mathrm{m}$ \\
Amarelo & $0,570-0,590 \mu \mathrm{m}$ \\
Laranja & $0,590-0,620 \mu \mathrm{m}$ \\
Vermelho & $0,620-0,750 \mu \mathrm{m}$ \\
\hline Fonte: Adaptado de Moraes $(2008)$
\end{tabular}

Os VANTs se beneficiam da variedade de sensores $R G B$ profissionais disponíveis no mercado, Colomina e Molina (2014) em sua revisão apresentaram uma lista das câmeras mais usadas em multirrotores e asas fixas (Figura 4). 


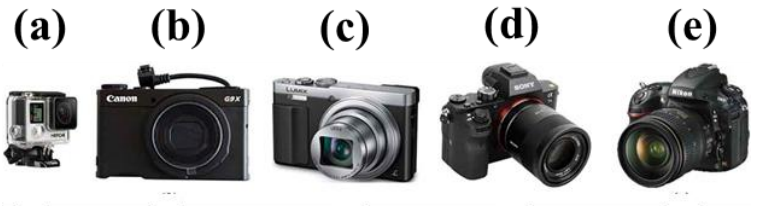

Fonte: adaptado de Pádua et al. (2017).

Figura 4 - Exemplos de câmeras $R G B$ comumente usadas em VANTs para aquisição de imagens RGB: (a) GoPro Hero 4 Black edition; (b) Canon G9X; (c) Panasonic Lumix DMC-TZ71; (d) Sony Alpha 7; e (e) Nikon D800.

\section{Sensores Infravermelhos}

Os sensores infravermelhos operam em uma região espectral que está situada entre $0,750 \mu \mathrm{m}$ (infravermelho próximo, NIR) e 1,0 $\mu \mathrm{m}$ (infravermelho distante, FIR). O NIR é encontrado principalmente na radiação solar ou fontes convencionais, e à medida que se aproxima do intervalo espectral do FIR observam-se características termais do alvo, provenientes da emissão eletromagnética de objetos terrestres (Moraes, 2008).

Normalmente, profissionais transformam sensores $R G B$, substituindo por um filtro $N I R$, removendo e substituindo-o por um que esteja filtrando as faixas vermelhas, verdes ou azuis visíveis; outra opção é a aquisição de sensores multiespectrais apresentados na Figura 5. (a)

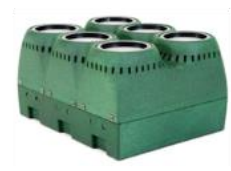

(b)

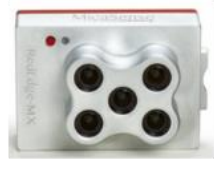

(c)

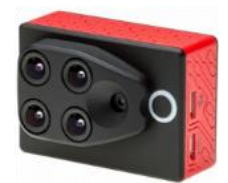

Figura 5 - Sensores multiespectrais no mercado: (a) Kernel 6 Camera Array; (b) RedEdge-MX ; (c) Parrot SEQUOIA+ Multispectral Sensor.

O infravermelho é bastante utilizado em práticas agrícolas, que necessitam uma análise precisa da vegetação. Quando combinado com comprimentos de ondas das bandas $R G B$ o NIR permite a exploração mais eficiente dos atributos da vegetação (Shahrokhnia e Ahmadi, 2019). A manipulação das bandas espectrais através das normalizações e expressões matemáticas possibilitaram novas características espectrais da vegetação, as quais são correlacionadas com parâmetros biofísicos da vegetação, por exemplo, vigor vegetativo ou índice de área foliar (Helbich, 2019).

\section{Sensores LiDAR}

A tecnologia dos sensores $L i D A R$ permite uma melhor precisão dos dados quando comparados aos sensores fotográficos. O sistema é baseado na transmissão de pulsos em forma de LASER em direção ao objeto na superfície terrestre com uma taxa de repetição rápida. Calculando o tempo do caminho realizado pelo pulso emitido (Sensoralvo-Sensor/ receptor) é possível determinar a distância e localização do alvo em seu plano cartesiano $\mathrm{x}$, y e $\mathrm{z}$ dos alvos, e, dessa forma, com o processamento das informações de retorno dos pulsos é gerado uma nuvem de pontos tridimensionais com maior exatidão e precisão (Giongo et al., 2012) (Figura 6). (a)

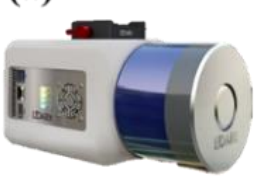

(b)

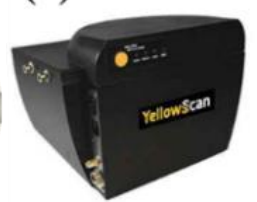

(c)

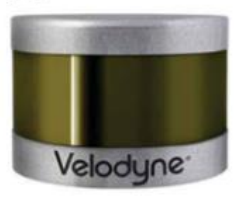

Figura 6 - Sensores LiDAR: (a) LiDARit Eagle X; (b) mapeador da Yellowscan; (c) Velodyne PUCK.

\section{PROCESSAMENTO DAS IMAGENS DE UM SENSOR FOTOGRÁFICO}

\section{Pré-processamento das imagens}

A melhoria da tecnologia integrada aos sensores remotos proporcionou uma elevada aquisição de dados que necessitam ser tratados para a extração de informações, dessa forma, reduzindo problemas que possam afetar a qualidade dos produtos (Geipel et al., 2013).

A variabilidade espectral é um fator essencial, que contribue para redução da qualidade da imagem, sendo imprencisdível realizar uma calibração radiométrica para que seja possível obter valores confiáveis sobre reflectâncias das imagens em função da iluminação e característica do sensor. (Jorge e Inamasu, 2014).

Para a calibração radiométrica é utilizado um alvo que possui referências fixas para cada banda a ser obtida pelo sensor multiespectral permitindo a comparação temporal entre levantamentos aéreos, realizados em dias com diferente insolação e condições climáticas (Kurihara et al., 2020) (Figura 7). 


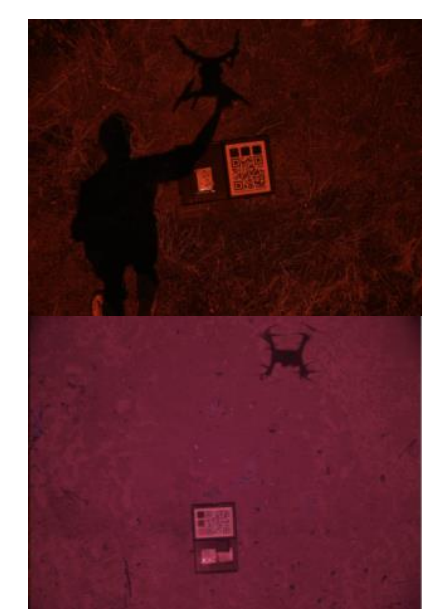

Fonte: Mapir camera (2020)

Figura 7. Alvo de calibração radiométrica

\section{Reconstrução tridimensional}

Ao realizar um levantameto aerofotogramétrico de uma área de estudo, na maioria dos casos, é necessário capturar várias imagens para cobrir o local de interesse o que torna imprescidível o alinhamento dessas fotografias por meio da execução do algoritmo structure from motion (SfM), que a partir de várias fotografias sobrepostas reconstrói a área em três dimensões (Verhoeven, 2011), sendo que, a partir dessa etapa é que haverá a extração de vários recursos por meio de imagens de um sensor fotogramétrico (Figura 8).

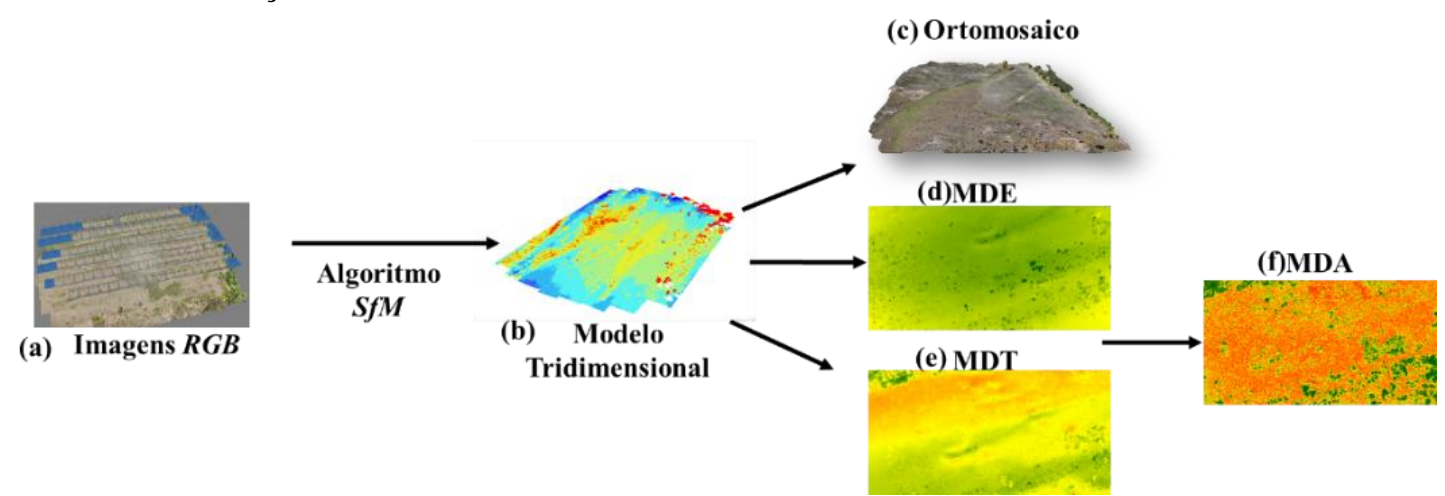

Figura 8 - Fluxograma do processo de geração dos produtos: (a) Imagens $R G B$; Modelo tridimensional; (c) Ortomosaico; (d) Modelo Digital de Elevação; (e) Modelo Digital de Terreno; (f) Modelo Digital de Altura, obtida por meio da subtração entre MDE e MDT.

O Processo pode ser subdividos em 4 etapas: (1) distribuição de pontos de controle (PC) na área e coleta das coordenadas com um receptor geodésico; (2) levantamento aéreofotogramétrico (Figura 9); (3) alinhamento das imagens com um software; (4) introdução das coodernadas (PC). O software utiliza estas coordenadas como referência, transportando estas coordenadas conhecidas para imagens, realizando $o$ georreferenciamento de cada pixel da imagem.

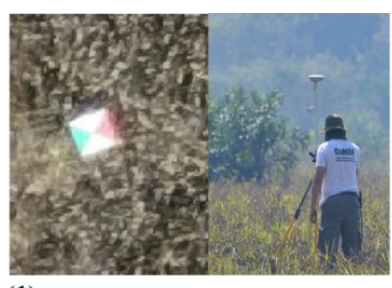

(1)

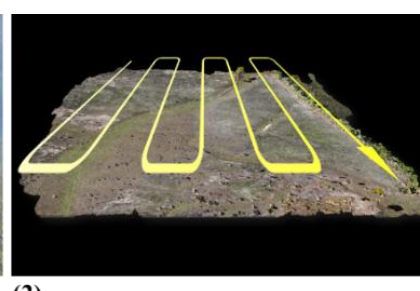

(2) integrado, responsável por executar missões aéreas, no entanto, a precisão planialtimétrica é considerada baixa, fazendo-se necessário o uso de um GPS de alta precisão para realizar as devidas correções (Meinen e Robinson, 2020).

\section{ÍNDICES DE VEGETAÇÃO (IVs)}

\section{Interação da Radiação Eletromagnética e Vegetação}

A característica intrínseca das propriedades espectrais das plantas é representada pelo valor de reflectância do alvo, que por sua vez, depende principalmente das particularidades morfológicas, estruturais e químicas da vegetação. Visto a importância em considerar a resposta espectral da vegetação para estudo da cobertura vegetal, é fundamental a compreensão das suas estruturas e dos processos relevantes na fotossíntese e respiração (Jensen, 2009).

As folhas são revestidas pela epiderme superior e inferior, que por sua vez, é revestida pela

A grande maioria dos VANTs possui um GPS 
camada de cutícula de característica hidrofóbica que dificulta a perda de água pela planta ocasionada por fatores climáticos externos, por exemplo, ventos e altas temperaturas (Lopes et al., 2018).

As trocas gasosas e saída de água são realizadas por pequenos poros situados na epiderme, denominados de estômatos, os quais são encontrados em maior quantidade na epiderme inferior (Taiz et al., 2017). Algumas folhas podem apresentar em sua estrutura externa tricomas, que, dependendo da sua estrutura, podem refletir parte da radiação eletromagnética, evitando o superaquecimento da planta (Ponzoni et al., 2015).

Abaixo dessas estruturas (cutícula e epiderme), encontra-se o mesófilo paliçádico, onde estão situadas organelas ricas em pigmentos fotossintetizantes (clorofila e carotenoides), responsáveis em absorver a radiação eletromagnética e desencadear o processo da fotossíntese (Taiz et al., 2017). O mesófilo esponjoso, encontrado na direção dorsal da folha, é caracterizado pela maior quantidade de espaço entre as células, onde são preenchidos por gases resultantes da respiração celular e transpiração (Vieira et al., 2010).

As estruturas de uma folha podem variar até mesmo entre espécies, o que pode influenciar as propriedades espectrais da planta. $\mathrm{O}$ mais importante é compreender o processo de interação da planta com a radiação eletromagnética, através da reflexão, transmissão e absorção.

As relações entre a reflectância, transmissão e absorção, são apresentadas na Figura 10, em um corte na seção transversal da folha representando possíveis caminhos da incidência da radiação eletromagnética na folha.

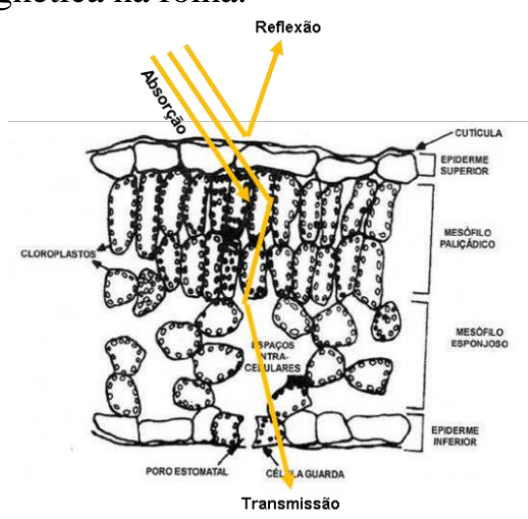

Fonte: Ponzoni et al. (2015)

Figura 10- Corte transversal de uma folha e possíveis caminhos da incidência da radiação eletromagnética.

Quando a radiação solar incide sobre uma planta, parte dessa radiação é refletida pelas estruturas externas da folha, por exemplo, cutículas e tricomas (Ponzoni et al., 2015). Outra fração da radiação eletromagnética é absorvida pela parte interna da folha, especialmente dentro dos cloroplastos, onde são encontrados pigmentos fotossintetizantes. A transmissão se dá após o processo de absorção, onde parte da radiação é transmitida para o dorso da folha (Mohammed et al., 2019).

Para desencadear o processo de fotossíntese a clorofila absorve a radiação eletromagnética no comprimento de onda $0,450 \mu \mathrm{m}$ (azul), 0,650 $\mu \mathrm{m}$ (vermelho) e 0,550 $\mu \mathrm{m}$ (verde) (Figura 11).

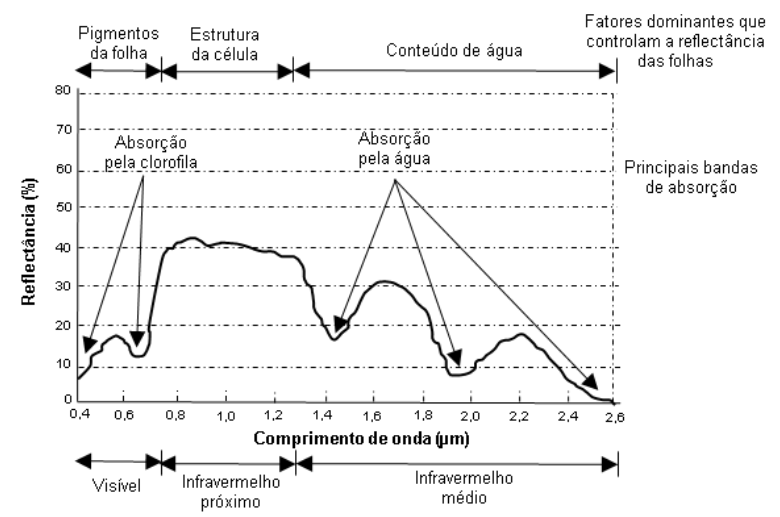

Fonte: Adaptado de Novo (2010)

Figura 11- Assinatura espectral de uma folha verde.

No comprimento de onda do infravermelho próximo $(0,72 \mu \mathrm{m})$ a absorção é considerada pequena, sendo refletida grande parte da radiação eletromagnética, resultado consequente da incidência de energia no mesófilo esponjoso e quanto mais espaçosa é a estrutura, maior será a reflectância (Novo, 2010). Situações de estresse e senescência da vegetação provocam mudanças na assinatura espectral da planta. Na Figura 12 são apresentadas duas situações comuns que uma vegetação pode apresentar.

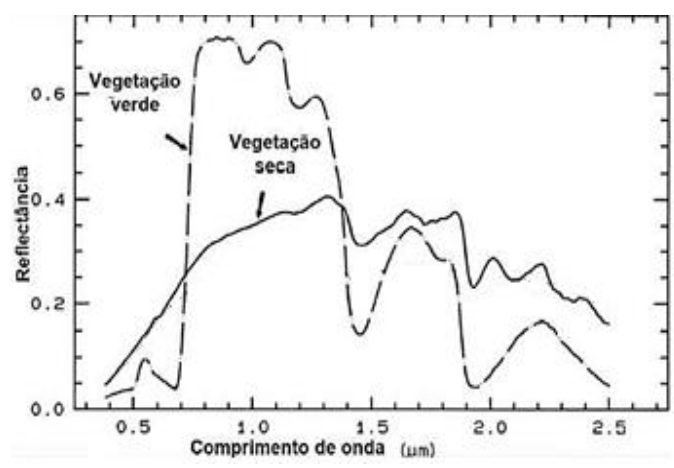

Fonte: Adaptado de Novo (2010)

Figura 12 - Assinatura espectral de uma vegetação em duas situações fisiológicas. 
$\mathrm{Na}$ vegetação verde a transição entre a região espectral do vermelho e do infravermelho próximo (borda do vermelho) é mais acentuada, expressando uma verticalidade em sua curva. Quando se observa uma vegetação seca há uma redução abrupta na taxa de reflectância e um aumento na faixa das ondas do comprimento visível (Novo, 2010).

\section{Índices de Vegetação (IVs)}

Para extrair informações espectrais de uma imagem, existe uma variedade de índices de vegetação que podem ser aplicados. Esses índices são calculados através do uso de informações sobre a refletância da superfície. Rosa (2009) ressalta que a maioria dos índices de vegetação utilizam as faixas espectrais do vermelho $\mathrm{e}$ infravermelho próximo, por conter a maior parte da variação espectral da planta, aplicando expressões de razões simples, por exemplo, cálculo de índice de diferença normalizada (NDVI), que permitem uma melhor compreensão do atual estado da vegetação. Uma diversidade de índices de vegetação baseados no teor de clorofila tem sido utilizada. Na Tabela 2, são apresentados os principais índices utilizados para estudos.

Tabela 2 - Índices de vegetação e suas respectivas expressões matemáticas.

\begin{tabular}{clc}
\hline Índice & Expressão & Referência \\
\hline NDVI & $\frac{N I R-R}{N I R+R}$ & (Rouse et al., 1974)
\end{tabular}

SAVI

$$
\frac{(1+L) *(N I R-R)}{N I R+R+L}
$$

(Huete, 2002)

GEMI

$$
\begin{gathered}
\frac{n(1-0,25 n)-(R-0,125)}{(1-R)} \\
n=\frac{2 *\left(N I R^{2}-R^{2}\right)+1,5 * N I R+0,5 * R}{(R+N I R+0,5}
\end{gathered}
$$

(Pinty e Verstraete, 1992)

Legenda: NDVI, índice de diferença normalizada; SAVI, índice de vegetação ajustado ao solo; GEMI, índice global de monitoramento $\mathrm{R}=$ vermelho; $\mathrm{NIR}=$ infravermelho próximo; $\mathrm{L}=0,25$ a 1 dependendo da cobertura do solo

O índice de diferença normalizada (NDVI) é um indicativo do estado da vegetação em relação ao seu vigor e densidade vegetativa, cujo valores variam entre -1 a 1 , sendo que o solo exposto e a hidrologia se aproximam dos valores negativos. Nos casos em que a presença do solo precisar ser levada em consideração, o índice de vegetação ajustado ao solo $(S A V I)$ realiza os devidos ajustes considerando um fator de correção que varia de 0,25 a 1 , valor variante conforme a cobertura do solo (Bendig et al., 2015).
O Índice de Monitoramento Global (GEMI) é uma expressão não linear, semelhante ao NDVI, no entanto, é menos sensível aos efeitos atmosféricos, sendo afetado principalmente pelo solo exposto, recomendado para áreas com densa cobertura vegetal (Pinty e Verstraete, 1992). Além dos índices usuais, algumas expressões têm sido propostas para analisar situações especificas, por exemplo, detecção de queimadas ou ausência do espectro do infravermelho (Tabela 3 ).

Tabela 3 - Outros índices de vegetações para situações específicas.

\begin{tabular}{ccc}
\hline Índice & Expressão & Referência \\
\hline NBR & $\frac{N I R-S W I R 2}{N I R+S W I R 2}$ & (Key e Benson, 1999) \\
ExG & $2 * G-R-B$ & (Li et al., 2016) \\
CIVE & $0,441 * R-0,881 * G+0,385 * B+18,78745$ & (Kataoka et al., 2003) \\
ExGR & $E x G-1,4 * R-G$ & (Li et al., 2016) \\
NGRDI & $\frac{G-R}{G+R}$ & (Gitelson et al., 2016) \\
\hline
\end{tabular}

Legenda: NBR, relação queimada normalizada; Exg, Excesso de verde; CIVE, índice de cores da vegetação; ExGR, Excesso de verde menos vermelho; NGRDI, índice de diferença normalizada verde - vermelho; NIR, infravermelho próximo; SWIR2, infravermelho médio; G, R e B, verde, vermelho e azul, respectivamente. 


\section{APLICAÇÕES DO USO DOS VANTs}

$\mathrm{O}$ uso dos sensores integrados aos VANTs permite aquisição de imagens aéreas de alta resolução, expandindo novas possibilidades de estudo da vegetação capazes de enfrentar tecnologias consolidadas no mercado (Miranda et al., 2020). Neste tópico serão abordados alguns trabalhos baseados em VANTs na área da silvicultura e incêndios florestais. Na área florestal as principais aplicações incluem o mapeamento do dossel da floresta e o inventário florestal. Em relação aos incêndios florestais, os estudos exploram a quantificação do material combustível, fator relevante para a propagação do fogo (Soares et al., 2017).

\section{Silvicultura}

Giongo et al. (2012) utilizaram dados laser scanning aerotranpostado (LiDAR) para estimar a altura da base das copas das árvores em uma área situada na cidade de Curitiba-PR, visando avaliar a potencialidade da varredura com laser. Os resultados mostraram-se bastante eficientes e com grande potencial, principalmente em áreas de difícil acesso, onde métodos convencionais seriam complexos e onerosos, concluindo que a difusão dos dados laser scanning poderá contribuir para o setor florestal.

Getzin et al. (2012) capturaram imagens aéreas de uma floresta por meio de um sensor fotográfico em um VANT de asa fixa, cujo propósito foi realizar um exame mais aprofundado dos dosseis e avaliar a biodiversidade florística existente no subbosque da floresta, levando os autores concluirem a pontencialidade das imagens em detectar pertubações nos padrões dos dosseis.

\section{Incêndios Florestais}

Os estudos relacionados aos incêndios florestais possuem uma séries de variáveis que influenciam nos modelos do comportamento do fogo como, por exemplo, o material combustível, que, segundo Soares et al. (2017), em qualquer incêndio florestal é necessário haver material combustível para manter o processo de combustão.

Souza et al. (2018) utilizaram imagens de um sensor $R G B$ acoplado em um multirrotor com a finalidade de estimar o material combustível por meio da modelagem matemática em área do cerrado campo sujo. Os resultados levaram os autores a concluírem a eficiência e a pontencialidade da ferramenta para estimar o material combustível. Andersen et al. (2005), apresentaram o uso de variáveis métricas obtidas por dados laser scanning para estimar combustível de copa, altura da base de copa, incluindo a massa do material combustível de copa e densidade de massa de copa em floresta de coníferas. Os resultados do estudo indicaram que o LiDAR pode ser utilizado para estimar as métricas do material do combustível do dossel de maneira eficiente e precisa em uma extensa área dentro de uma floresta de coníferas do noroeste do Pacífico.

\section{Recomendações do Uso de sensores acoplados em VANTs}

A Tabela 4 contém informações adaptadas de Pádua et al. (2017) sobre o uso de sensores e VANTs em função da área de aplicação e área de cobertura. Os VANTs baseados em multirrotores são mais adequados em levantamento de áreas pequenas, enquanto os asas fixas são aplicados em áreas extensas.

Tabela 4 - Sensores e VANTs para diferentes aplicações.

\begin{tabular}{cccc}
\hline Área de aplicação & Área de cobertura & $\begin{array}{c}\text { Sensor (es) } \\
\text { recomendado (s) }\end{array}$ & $\begin{array}{c}\text { UAV } \\
\text { recomendado }\end{array}$ \\
\hline Estimativa de biomassa & Ampla & $\begin{array}{c}\text { Óptica } \\
\text { Óptica }\end{array}$ & $\begin{array}{c}\text { Asa fixa } \\
\text { Multirrotor }\end{array}$ \\
\hline Mequena & Ampla & LiDAR & Asa fixa \\
\hline Inventontário florestal & Ampla & LiDAR & Asa fixa \\
\hline $\begin{array}{c}\text { Medição e monitoramento de propriedades } \\
\text { florestais estruturais }\end{array}$ & Ampla & LiDAR & Asa fixa \\
\hline Detecção e monitoramento de incêndios florestais & Ampla & Térmico & Asa fixa \\
\hline Estimativa da área de queima após o incêndio & Ampla & Multiespectral & Asa fixa \\
\hline Mapas da altura da vegetação & Pequena & Óptica & Multirrotor \\
\hline
\end{tabular}


A escolha do sensor que será integrado ao VANT dependerá principalmente do tipo de aplicação, conforme detalhado na Tabela 4 . Comumente os sensores baseados em laser scanning são os mais indicados para aplicações florestais, onde a alta densidade de copa dificulta a penetração da luz. Situações onde há necessidade de determinar áreas queimadas os sensores multiespectrais e térmicos são os mais recomendáveis.

Uma alternativa para se obter estimativa de biomassa e mapeamento da altura da vegetação é a utilização de sensores ópticos que operam na faixa espectral do visível, sendo uma potencial tecnologia em relação ao seu custo benefício quando comparada ao LiDAR, cujas as imagens podem ser processadas facilmente pelo algoritmo SfM.

\section{REFERÊNCIAS BIBLIOGRÁFICAS}

Andersen HE, Mcgaughey RJ, Reutebuch SE. Estimating forest canopy fuel parameters using LIDAR data. Remote Sensing of Environment, v.94, n.4, p.441-449, 2005.

Bendig J, Yu K, Aasen H, Bolten A, Bennertz S, Broscheit J, Gnyp LM, Bareth G. Combining UAV-based plant height from crop sufarce models, visible and near infrared vegetation indices for biomass monitoring in barley. International Journal of Applied Earth Observation and Geoinformation, v.39, p.79-87, 2015.

http://doi.org/10.1016/j.jag.2015.02.012.

Colomina I, Molina P. Unmanned aerial systems for photogrammetry and remote sensing: A review. ISPRS Journal of Photogrammetry and Remote Sensing, v.92, p.79-97, 2014.

https://doi.org/10.1016/j.isprsjprs.2014.02.013.

Geipel J, Peteinatos GG, Claupein W, Gerhards R. Enhancement of Micro Unmanned Aerial Vehicles for Agricultural Aerial Sensor Systems. In Precision Agriculture '13, Ed.J. V. Stafford, p. 161-167. Wageningen, the Netherlands: Wageningen Academic Publishers, 2013.

Getzin S, Wiegand K, Schöning I. Assessing biodiversity in forests using very high-resolution images and unmanned aerial vehicles. Methods in Ecology and Evolution, v.3 n.2, p.397-404, 2012.

https://doi.org/10.1111/j.2041-210X.2011.00158.x

Giongo M, Koehler HS, Viola MR, Bastos OS, Santos AF, Santopuoli G. Estimativa da altura da base das copas com o uso de dados laser scanning aerotransportado (LiDAR). Journal of Biotechnology and Biodiversity, v.3, n.3, p.48$57,2012$.

https://doi.org/10.20873/jbb.uft.cemaf.v3n3.giongo

Gitelson AA, Kaufman JY, Stark R, Rundquist D. Novel algorithms for remote estimation of vegetation fraction. Remote Sensing of Environment, v.80, n.1, p.76-87, 2016. https://doi.org/10.1016/S0034-4257(01)00289-9

Hardin PJ, Jensen RR. Veículos aéreos não tripulados de pequena escala em sensoriamento remoto ambiental: desa- fios e oportunidades". GIScience e sensoriamento remoto, v.48, n.1, p.99-111, 2011.

https://doi.org/10.2747 / 1548-1603.48.1.99

Helbich M. Spatiotemporal Contextual Uncertainties in Green Space Exposure Measures: Exploring a Time Series of the Normalized Difference Vegetation Indices. Int. J. Environ. Res. Public Health, v.16, n.5, p.852, 2019. https://doi.org/10.3390/ijerph16050852

Huete AR, Didan K, Miura T, Rodriguez PE, Gao X, Ferreira LG. Overview of the radiometric and biophysical performance of the MODIS vegetation indices. Remote Sensing of Environment, v.83, n.1, p.195-213, 2002. https://doi.org/10.1016/S0034-4257(02)00096-2

Jensen JR. Sensoriamento Remoto do Ambiente: Uma perspectiva em recursos terrestres. São José dos Campos, SP: Parêntese, 2009.

Jorge LAC, Inamasu RY. Uso de veículos aéreos não tripulados (VANT) em agricultura de precisão. Embrapa Instrumentação-Capítulo em livro científico (ALICE), 2014.

Kataoka T, Kaneko T, Okamoto H, Hata S. Crop growth estimation system using machine vision. Proceedings 2003 IEEE/ASME International Conference on Advanced Intelligent Mechatronics (AIM 2003), v.2, n.1, p.1079-1083, 2003.

http://doi.org/10.1109/AIM.2003.1225492

Key CH, Benson NC. Measuring and remote sensing of burn severity. In: Neuenschwander LF, Ryan KC. Proceedings Joint Fire Science Conference and Workshop. Moscow: 3ed. University of Idaho and International Association of Wildland Fire. 1999, p. 284.

Kurihara J, Ishida T, Takahashi Y. Unmanned Aerial Vehicle (UAV)-based hyperspectral imaging system for precision agriculture and forest management. In Unmanned Aerial Vehicle: Applications in Agriculture and Environment (p. 25-38). Springer, Cham, 2020

Li W, Niu Z, Chen H, Li D, Wu M, Zhao W. Remote estimation of canopy height and aboveground biomass ofmaize using high-resolution stereo images from a lowcostunmanned aerial vehicle system. Ecological Indicator, v.67, n.1, p.637-648, 2016. http://dx.doi.org/10.1016/j.ecolind.2016.03.036

Liu WTH. Aplicações de sensoriamento remoto. Campo Grande: Oficina de Textos, 2015. 908p.

Lopes BA, Silva MM. Estratégias funcionais de Vanilla chamissoni (Orchidaceae) em ambiente arbustivo e florestal de restinga. Revista Brasileira de Geografia Física, v.12, n. 2 , p. 355-364, 2018

https://doi.org/10.26848/rbgf.v12.2.p355-364

Mapir camera. Calibration Target (V2) capture Procedure. 2020. Disponível em: < https://www.mapir.camera/pages/calibration-targetcapture-procedure-v2/>. Acesso em: 20 fev. 2020.

Meinen BU, Robinson DT. Mapping erosion and deposition in an agricultural landscape: Optimization of UAV image acquisition schemes for SfM-MVS. Remote Sensing of Environment, v.239, n.111666, 2020. https://doi.org/10.1016/j.rse.2020.111666

Miranda V, Pina P, Heleno S, Vieira G, Mora C, Schaefer CE. Monitoring recent changes of vegetation in Fildes Pen- 
insula (King George Island, Antarctica) through satellite imagery guided by UAV surveys. Science of The Total Environment, v.704, p.135295, 2020. https://doi.org/10.1016/j.scitotenv.2019.135295

Mohammed HG, Colombo R, Middleton E, Rascher U, Van Der Tol C, Nedbal L, Goulas Y, Pérez - Priego O, Damm A, Meroni M, Joiner J, Cogliati S, Verhoef W, Malenovsky Z, Gastellu - Etchegorry JF, Miller J, Guanter L, Moreno J, Moya I, Berry JA, Frankenber C, Zarco Tejada PJ. Remote sensing of solar-induced chlorophyll fluorescence (SIF) in vegetation: 50 years of progress. Remote Sensing of Environment, v.231, n.1, p.1-38, 2019. https://doi.org/10.1016/j.rse.2019.04.030

Moraes CE. Formação continuada de professores: curso de astronáutica e ciências do espaço. São Paulo: Editado pelo programa AEB Escolada Agência Espacial Brasileira, 2008. 78p.

Novo EML. Sensoriamento Remoto: princípios e aplicações. São Paulo: Edgard Blucher, 2010. 388p.

Pádua L, Vanko J, Hruška J, Adão T, Sousa JJ, Peres E, Morais R. UAS, sensors, and data processing in agroforestry: A review towards practical applications. International journal of remote sensing, v.38, n.8-10, p.2349-2391, 2017. https://doi.org/10.1080/01431161.2017.1297548

Pinty B, Verstraete M. GEMI - A nonlinear index to monitor global vegetation from satellites. Vegetation, v.101, n.1, p.15-20, 1992.

Ponzoni JF. et al. Sensoriamento remoto da vegetação. 2. ed. Atualizada e ampliada, São Paulo, SP: Oficina de Textos, 2015. 45p.

Przybilla HJ, Wester-Ebbinghaus W. Bildflug mit ferngelenktem Kleinflugzeug. Bildmessung und Luftbildwesen, v.47, n.5, p.137-142, 1979.

Raj R, Kar S, Nandan R, Jagarlapudi A. Precision Agriculture and Unmanned Aerial Vehicles (UAVs). In: Avtar R., Watanabe T. (eds) Unmanned Aerial Vehicle: Applications in Agriculture and Environment. Springer, Cham.

Rosa R. Introdução ao Sensoriamento Remoto 7.ed. Uberlândia: EDUFU, 2009. 264p.

Rouse W, Haas HR, Schell AJ, Deering WD. Monitoring vegetation systems in the Great Plains with ERT. Washington, NASA: third earth resources technology Satellite-1 symposium, 1 ed. 1974, 309p.

Shahrokhnia HM, Ahmadi HS. Remotely Sensed Spatial and Temporal Variations of Vegetation Indices Subjected to Rainfall Amount and Distribution Properties. Spatial Modeling in GIS and R for Earth and Environmental Sciences, v.1, n.1, p.21-53, 2019. https://doi.org/10.1016/B978-0-12-815226-3.00002-8

Simelli I, Tsagaris A. The Use of Unmanned Aerial Systems (UAS) in Agriculture. In HAICTA(p. 730-736).

Soares VR. et al. Incêndios florestais. Curitiba: UFPR, 2017. $255 \mathrm{p}$.

Souza VI, Santos MM, Giongo M, Carvalho VE, Machado SEI. Estimativa do material combustível em área de cerrado campo sujo a partir de imagens do sensor RGB. Pesqui- sa Florestal Brasileira, v.3, n.1, p.01-06, 2018. https://doi.org/10.4336/2018.pfb.38e201801706

Taiz L, Zeiger E, Moller MI, Murphy A. Fisiologia e Desenvolvimento Vegetal. 6. ed. Porto Alegre, RS: ARTMED EDITORA LTDA, 2017. 731p.

Verhoeven G. Taking computer vision aloft - Archaeological three-dimensional reconstructions from aerial photographs with PhotoScan. - Archaeological Prospection, v.18, n.1, p.67-73, 2011.

Vieira LE, Souza SG, Santos RA, Silva S.J. Manual de fisiologia vegetal. 1. ed. São Luis, MA: EDUFMA, 2010. 230p. 